\title{
Differential responses of cellular immunity in patients undergoing neoadjuvant therapy followed by surgery for carcinoma of the oesophagus
}

\author{
Marinke Westerterp • Marja A. Boermeester · Jikke M. T. Omloo • \\ Maarten C. C. M. Hulshof - Walter L. Vervenne - Rene Lutter • Theo A. Out • \\ J. Jan B. van Lanschot
}

Received: 22 August 2007 / Accepted: 25 March 2008 / Published online: 9 April 2008

(C) The Author(s) 2008

\begin{abstract}
Background To compare immune responses following neoadjuvant chemoradiation therapy in combination with hyperthermia plus surgery to those induced by surgery alone in patients with oesophageal cancer.

Methods Thirty-two patients with histopathologically proven oesophageal cancer, scheduled for potentially curative transhiatal or transthoracic oesophagectomy with (neo, $n=20$ ) or without (control, $n=12$ ) neoadjuvant thermochemoradiation therapy (ThCR) were included. Peripheral blood samples were obtained before ThCR, after 2 weeks of ThCR, 1 day before surgery, on postoperative days 1, 3, 7 , and 6 weeks after surgery, for white blood cell counts, lymphocyte subsets and T helper type 1 (Th1) and type 2 (Th2) lymphocyte responses.
\end{abstract}

M. Westerterp $(\square) \cdot$ M. A. Boermeester .

J. M. T. Omloo · J. J. B. van Lanschot

Department of Surgery, Academic Medical Centre,

Meibergdreef 9, 1105 AZ Amsterdam, The Netherlands

e-mail: mwesterterp@hotmail.com

M. C. C. M. Hulshof

Department of Radiotherapy,

Academic Medical Centre, Amsterdam, The Netherlands

W. L. Vervenne

Department of Medical Oncology,

Academic Medical Centre, Amsterdam, The Netherlands

R. Lutter · T. A. Out

Department of Experimental Immunology,

Academic Medical Centre, Amsterdam, The Netherlands

R. Lutter

Department of Pulmonology, Academic Medical Centre, Amsterdam, The Netherlands
Results Neo patients showed a significant decrease in granulocytes and lymphocyte subsets, and $\mathrm{T}$ cell cytokines after 2 weeks of ThCR. Only CD8+ (cytotoxic) T cells recovered after ThCR to reach normal levels prior to surgery. In contrast, CD4+ T (helper) cells, and NK- and B cells in neo patients did not recover prior to surgery (all $P<0.05)$. Oesophagectomy induced a significant increase in granulocytes and a decrease in lymphocytes (and subsets). Only those subsets that had not recovered after ThCR (CD4+ T cells, NK and B cells but not CD8+ T cells), were significantly lower (all $P<0.05$ ) during the entire postoperative study period. Postoperatively, the stimulated cytokine production capacity of Th1 and Th2 cells, corrected for number of $\mathrm{T}$ cells, was not significantly different between the groups.

Conclusion Neoadjuvant thermochemoradiation for oesophageal cancer caused significant disturbances of host cellular immunity with reduced T, NK and B cell counts, and differential recovery of cytotoxic and helper $\mathrm{T}$ cells leading to prolonged $\mathrm{T}$ cell imbalance that extends beyond the time of surgery. The functional and anti-tumour consequences of this immunodisturbance need further investigation, as recovery of $\mathrm{T}$ helper cytokine production towards surgery was less impaired than $\mathrm{T}$ helper cell counts.

Keywords Oesophageal cancer - Neoadjuvant therapy · Surgery $\cdot$ Immune response $\cdot T$ cell imbalance

\section{Introduction}

Oesophageal cancer is associated with unfavourable prognosis. Surgical resection is the best option when curation is aimed for. However, even after surgery with curative intent, overall survival is only $20-40 \%[9,26]$. Many institutes 
apply neoadjuvant therapy to improve the long-term outcome, especially after the publication of favourable longterm results of a randomised trial from the Medical Research Council Oesophageal Cancer Working Party, comparing neoadjuvant chemotherapy followed by surgery versus surgery alone [23]. However, in a large proportion of patients insufficient objective response is achieved. To improve these disappointing response rates, different protocols of preoperative chemoradiation therapy have been proposed and evaluated [17]. Promising results have been achieved with the addition of heat to chemotherapy or radiotherapy. Hyperthermia is a treatment modality in which oesophageal tissue is exposed to high temperatures to damage and kill cancer cells, or to make cancer cells more sensitive to the effects of radiation and certain anticancer drugs. In the Academic Medical Centre in Amsterdam (the Netherlands), an external heating system has been developed using the AMC $70 \mathrm{MHz}$ four antenna array [8]. To evaluate the additional value of hyperthermia, a phase II study was initiated, combining radiochemotherapy plus deep regional hyperthermia (thermochemoradiation therapy = ThCR) as neoadjuvant treatment in operable oesophageal cancer patients.

Postoperative alterations in host immune functions after major surgical interventions have been extensively described and investigated [5, 6, 13]. A previous study by our group, investigating the alterations in immune responses after limited transhiatal oesophagectomy (THO) and extended transthoracic oesophagectomy (TTO), demonstrated that both THO and TTO severely suppressed $\mathrm{T}$ helper type-1 (Th1) and type-2 (Th2) host immune responses. The extent of the surgical procedure had a differential immunosuppressive impact on Th2 cell activity (Th2 production of IL-4 and IL-13) but not on Th1 cell activity (Th1 production of IL-2 and IFN- $\gamma$ ). The two operations similarly impaired $\mathrm{T}$ helper type 1 cytokine production, which indicates that the two $\mathrm{T}$ helper pathways were down regulated through distinct mechanisms [42]. The addition of preoperative thermochemoradiation therapy may suppress the immunological state of the patients even to a greater extent than oesophagectomy alone, but only limited data are available on immune responses after neoadjuvant therapy followed by surgery $[12,20]$. Furthermore, in the interval between neoadjuvant therapy and surgery recovery may take place, which is an important prerequisite for immunocompetence during and after surgery. The host recovery capacity and extent of recovery in the neoadjuvant setting is not well known.

Therefore, the objective of this study was to compare immunological parameters in patients with oesophageal cancer subjected to curative surgery with and without ThCR. Our analysis focused on the kinetics and recovery of lymphocyte subsets as well as stimulated Th-cell cytokine production that reflects the critical balance between Th1and Th2-mediated immune responses.

\section{Methods}

Patients

Between January 2004 and January 2005, 32 patients with histologically proven cancer of the oesophagus or oesophagogastric junction consented to participate in the present study. All patients were scheduled for a potentially curative oesophagectomy by either a limited transhiatal or an extended transthoracic approach. Twenty patients underwent neoadjuvant therapy prior to surgery (neo patients) and 12 patients (control patients) underwent a potentially curative oesophagectomy without preoperative therapy. Patients younger than 75 years could participate in the prospective nonrandomised trial to undergo neoadjuvant therapy if there was less than $2 \mathrm{~cm}$ gastric involvement. Other inclusion criteria were written informed consent and mentally, physically, and geographically able to undergo treatment and follow-up. Exclusion criteria were diabetes; uncontrolled heart failure, hypertension, severe arrhythmia, and pacemaker; pre-existing myelopathy and/or polyneuropathy; previous radiotherapy and/or chemotherapy; age $>75$ years and $<18$ years.

\section{Neoadjuvant therapy}

Neoadjuvant therapy consisted of a 5-week schedule of ThCR. The schedule was as follows: chemotherapy, consisting of paclitaxel $50 \mathrm{mg} / \mathrm{m}^{2}$ and carboplatin $\mathrm{AUC}=2$, by intravenous infusion on days 1, 8, 15, 22 and 29; radiotherapy consisting of a total of $41.4 \mathrm{~Gy}$, given in 23 fractions of $1.8 \mathrm{~Gy}, 5$ fractions per week, starting day 1 of the first cycle of chemotherapy; and hyperthermia with concurrent chemotherapy administration within $1 \mathrm{~h}$ after the radiotherapy treatment. The hyperthermia method has previously been described in detail by Albregts et al. [1]. Four to 6 weeks after the end of neoadjuvant therapy, patients were scheduled for surgery.

Histopathological evaluation

All specimens were evaluated by an experienced gastrointestinal pathologist, in accordance with the criteria of the International Union Against Cancer, including stage, $\mathrm{R}$ classification and grade. Tumours were classified as histopathologically responding when less than $10 \%$ viable tumour tissue was found in the tumour bed; otherwise the tumour was classified as histopathologically non-responding [44]. The group of non-responders was further divided into 
patients with partial response (10-50\% viable residual tumour tissue), minimal response ( $>50 \%$ viable residual tumour tissue), and no change (absence of any regressive changes). In the group of responders, response was classified as complete (histological fibrosis with no viable residual tumour cells) and subtotal response $(<10 \%$ viable residual tumour cells) [21].

\section{Blood sampling}

Peripheral venous blood samples were obtained before neoadjuvant therapy, after 2 weeks of the start of neoadjuvant therapy, 1 day before surgery, on postoperative days $1,3,7$, and 6 weeks after surgery (in total 7 sampling time points). In control patients, blood was drawn at the same time points except for the samples taken before and during neoadjuvant therapy (a total of 5 sampling time points).

\section{Leucocytes and differential counts}

The Beckman Coulter ACT Diff Analyser (Global Medical Instrumentation, Inc., Ramsey, MN, USA) was used for leucocyte and differential counts in peripheral venous blood samples, collected in ethylenediamine tetra-acetic acid (EDTA) tubes.

\section{Flow cytometry}

Peripheral venous blood samples were collected in EDTA collection tubes. The monoclonal antibodies used for immunophenotyping were from Becton Dickinson (San Jose, CA, USA). Multitest reagents were used in combination with True Count Tubes and FACS Lysing Solution (all Becton Dickinson), according to the instructions of the manufacturer. The antibody combination CD3CD8CD45 CD4 or CD3CD16CD56CD45CD19 was applied: antibody CD45 for lymphocytes, CD3 for T lymphocytes, CD3CD4 for $\mathrm{T}$ helper lymphocytes, CD3CD8 for T cytotoxic lymphocytes, CD3CD16CD56 for natural killer cells and CD19 for B lymphocytes.

Flow cytometric analysis was performed on an FACS Calibur flow cytometer (Becton Dickinson) using the Multiset software package (Becton Dickinson). A minimum of 2,000 and 5,000 lymphocytes were measured for T cell and for NK and B cell analysis, respectively.

Whole blood cultures

Whole heparin blood was diluted 1:10 in Iscove's modified Dulbecco's medium (IMDM; Boehringer Ingelheim, Alkmaar, The Netherlands), supplemented with $0.1 \%$ faetal calf serum, penicillin $(100 \mathrm{IU} / \mathrm{ml})$, streptomycin $(100 \mu \mathrm{g} / \mathrm{ml})$, and $15 \mathrm{IU} / \mathrm{ml}$ sodium heparin (Leo Pharmaceutical Prod- ucts, Weesp, The Netherlands) and was cultured in triplicate in flat bottom 200- $\mu$ l wells (Nunc, Roskilde, Denmark). In order to assay the stimulation of cytokines, diluted whole blood was stimulated with anti-CD3 (CLBT3/4.E, $100 \mathrm{ng} / \mathrm{ml}$ ) in concert with anti-CD28 (CLB-CD28/ $1,1 \mu \mathrm{g} / \mathrm{ml}$; Sanquin, Amsterdam, The Netherlands) for cross-linking of T cell CD3 and CD28 receptors. Supernatants were harvested after $24 \mathrm{~h}$ (for IL-2) and after $72 \mathrm{~h}$ (for IFN- $\gamma$, IL- 4 , and IL-13) and then stored at $-80^{\circ} \mathrm{C}$ until analyses.

Cytokine assays

Supernatant cytokine levels were measured using appropriate combinations of singleplex assays from Bio-Rad (Veenendaal, The Netherlands). Supernatant was diluted 1 in 4, using Bio-Plex human serum diluent, and fluorescence signals were analysed on the Bio-Plex reader after calibration with the high calibration setting. Cytokine production per $\mathrm{T}$ cell was calculated by dividing the amount of each cytokine $(\mathrm{pg} / \mathrm{ml})$ by the number of $\mathrm{CD} 3+$ cells.

\section{Statistical analysis}

All data were analysed using Statistical Software Package version 12.0.2 (SPSS Inc., Chicago, IL, USA) for Windows XP. Comparisons between groups were made using the Mann-Whitney test (for continuous variables). To determine the effect of neoadjuvant therapy on immune responses, the Wilcoxon rank test was applied to compare values 2 weeks after neoadjuvant therapy to those at baseline. To determine the effect of surgery on immune responses, the Wilcoxon rank test was applied to compare values of days after operation to those at baseline within the group. Between-group differences over time were analysed by a general linear model for repeated measures. By checking, residuals were approximately normally distributed. Standard descriptive analysis was performed for values at baseline. In figures, data are presented as mean values \pm one standard error of the mean. Statistical significance was defined as $P<0.05$ (two-sided).

\section{Results}

Clinicopathological data

Demographic characteristics, histopathological tumour indices and treatment-related complications were equally distributed among neo patients and control patients (Table 1).

Neo patients. The median patient age was 60 years (range $30-73$ years) and most patients were male $(90 \%)$. One patient developed a mediastinitis due to neoadjuvant therapy. 
Table 1 Clinicopathological parameters

\begin{tabular}{llll}
\hline & Neo $(N=20)$ & Control $(N=12)$ & $P$ value* \\
\hline Age (years) & $60(30-73)$ & $65(49-75)$ & NS \\
Gender (M:F) & $18: 2$ & $9: 3$ & NS \\
$\begin{array}{l}\text { cTNM stage of } \\
\quad \text { disease (I/II:III/IV) }\end{array}$ & $0 / 7: 13 / 0$ & $0 / 4: 7 / 1$ & NS \\
SCC:AC & $6: 14$ & $2: 10$ & NS \\
TTO:THO: no resection & $15: 4: 1$ & $6: 6: 0$ & NS \\
$\begin{array}{l}\text { Treatment related } \\
\quad \text { complications }\end{array}$ & 7 & 3 & NS \\
$\quad$ Mediastinitis & 1 & & \\
Anastomotic leakage & 3 & 1 & \\
Pneumonia & 3 & 1 & \\
Brain haemorrhage & & 1 &
\end{tabular}

Continuous variables are expressed as medians and ranges

Neo patients with preoperative treatment, control patients without preoperative treatment, tumour metastasis, $S C C$ squamous cell carcinoma, $A C$ adenocarcinoma, TTO extended transthoracic oesophagectomy, THO limited transhiatal oesophagectomy

* Mann-Whitney test (continuous variables) or Fisher exact test (categorical variables)

During early postoperative period (until discharge from the hospital), the following complications occurred (in 35\% of the patients): anastomotic leakage $(n=3)$ and pneumonia $(n=3)$.

Control patients. The median age was 65 years (range 49-75 years), and most patients were male (75\%). During early postoperative period complications were observed in $25 \%$ of patients. One patient had anastomotic leakage; one patient developed pneumonia, another patient suffered from brain haemorrhage.

\section{Histopathological response}

Histopathological response evaluation revealed complete response in 3 of the 20 neo patients (15\%), subtotal response in 6 patients $(30 \%)$, partial response in 4 patients (20\%), minimal response in 6 patients $(30 \%)$, and no change in 1 patient (5\%). There was no significant difference in immune response between responders and nonresponders to thermochemoradiation therapy.

Parameters of cellular immunity at baseline, during neoadjuvant therapy, and prior to surgery

\section{White blood cell and differential counts}

Baseline values (values before ThCR for neo patients, and values before operation for control patients) of white blood cell (WBC) and differential cell counts were similar in neo and control patients (Table 2).
Table 2 Baseline values of leucocyte and lymphocyte subsets

\begin{tabular}{llll}
\hline & Neo $(N=20)$ & Control $(N=12)$ & $P$ value* \\
\hline $\begin{array}{l}\text { Leucocytes } \\
\text { Differential counts }\end{array}$ & $6.7(5.1-15.0)$ & $7.7(1.2-11.7)$ & 0.851 \\
Granulocytes & $4.6(2.7-12.3)$ & $4.8(0.5-6.9)$ & 1.000 \\
Monocytes & $0.4(0.01-1.8)$ & $0.4(0.2-2.1)$ & 0.346 \\
Lymphocytes CD45+ & $1.8(1.1-2.7)$ & $2.2(1.1-3.8)$ & 0.350 \\
Lymphocyte subsets & & & \\
T lymphocytes (CD3+) & $1.4(0.7-2.0)$ & $1.6(0.7-2.4)$ & 0.497 \\
T helper (CD3+CD4+) & $0.8(0.2-1.1)$ & $1.0(0.6-1.8)$ & 0.112 \\
T cytotoxic (CD3+CD8+) & $0.4(0.2-1.1)$ & $0.4(0.1-0.7)$ & 0.611 \\
B cells (CD19+) & $0.2(0.05-0.4)$ & $0.2(0.05-0.6)$ & 0.134 \\
NK cells & $0.2(0.08-0.4)$ & $0.3(0.07-0.6)$ & 0.276 \\
\multicolumn{1}{l}{ CD3-CD16+CD56+) } & & & \\
\hline
\end{tabular}

Continuous variables are expressed as medians and ranges $\left(\times 10^{9} / 1\right)$ before neoadjuvant therapy (neo) and before operation (control)

$N K$ natural killer cells

* Mann-Whitney test (continuous variables)

After 2 weeks of ThCR, leucocytes, granulocytes (Fig. 1), lymphocytes (Fig. 2), and monocytes decreased significantly in all neo patients as compared to pre-treatment baseline values $(P<0.001, P=0.001$ and $P=0.007$, respectively). In the period between ThCR and operation, there was a trend to recovery in leucocytes, granulocytes and monocytes, although baseline values were not reached (leucocytes: $P=0.004$, monocytes: $P=0.255$, data not shown, granulocytes: $P=0.03$, Fig. 1). Lymphocyte values increased significantly towards operation as compared to the value after 2 weeks of ThCR $(P=0.002)$ but did not reach baseline values $(P<0.001$, Fig. 2$)$.

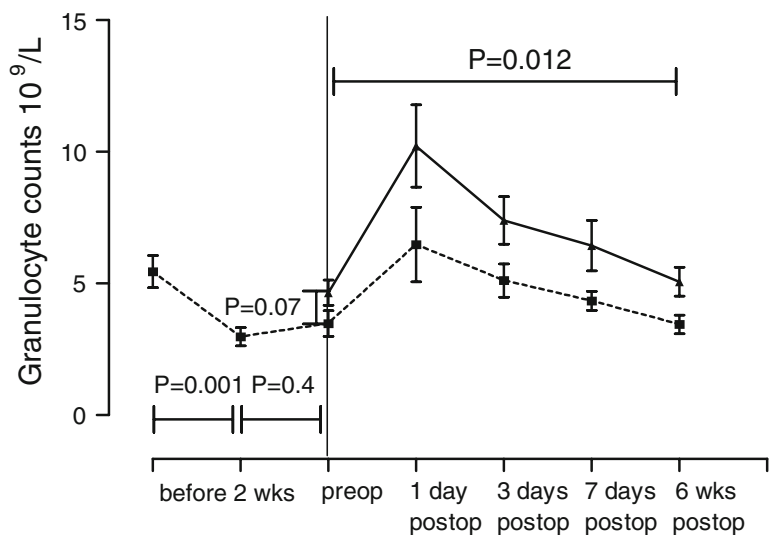

Fig. 1 Granulocyte counts: patients with oesophageal cancer treated with (dashed line with filled square, neo) and without (solid line with filled triangle, controls) preoperative thermochemoradiation therapy. Values after 2 weeks of neoadjuvant therapy versus baseline values: $P=0.001$. Preoperative values versus values after 2 weeks of neoadjuvant therapy, $P=0.4$; neo versus controls, 1 day preoperatively, $P=0.07$. Between-group differences over time in the 6 weeks postoperative period; $P=0.012$ 


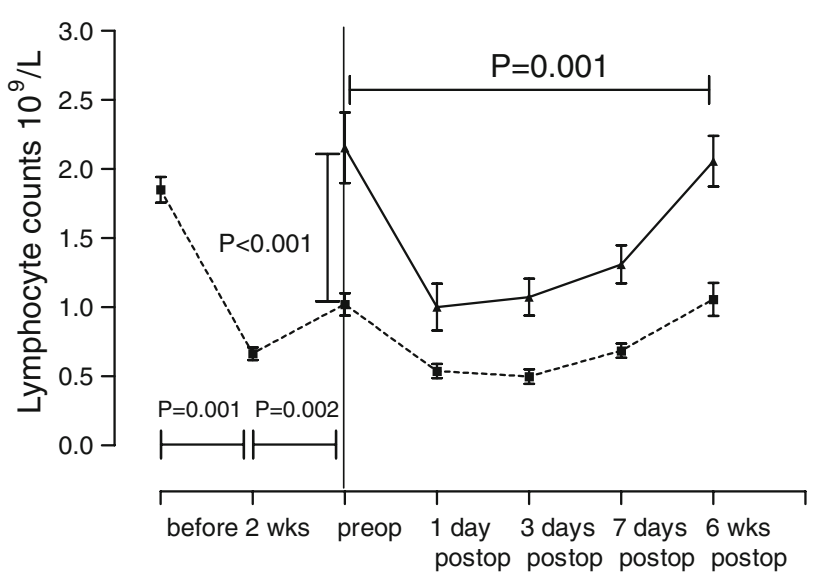

Fig. 2 Lymphocyte counts: patients with oesophageal cancer treated with (dashed line with filled square, neo) and without (solid line with filled triangle, controls) preoperative thermochemoradiation therapy. Values after 2 weeks of neoadjuvant therapy versus baseline values: $P<0.001$. Preoperative values versus values after 2 weeks of neoadjuvant therapy, $P=0.002$; neo versus controls, 1 day preoperatively, $P<0.001$. Between-group differences over time in the 6 weeks postoperative period; $P<0.001$

Preoperatively, leucocytes and lymphocytes (Fig. 2) were significantly lower in neo patients as compared to control patients $(P=0.004$ and $P<0.001$, respectively). This was in contrast to granulocytes (Fig. 1) and monocytes which were not significantly different between both groups at that time point $(P=0.07$ and $P=0.28$, respectively).

\section{Lymphocyte subsets}

Baseline values of lymphocyte subsets were similar in neo and control patients (Table 2).

After 2 weeks of ThCR, all lymphocyte subsets had decreased significantly (Figs. 3, 4, 5, 6). In the period between ThCR and operation, neo patients showed a significant recovery in CD16+CD56+ NK cells $(P=0.001$, Fig. 5) and $\mathrm{CD} 3+\mathrm{T}$ cells $(P=0.006$, data not shown $)$. Remarkably, recovery of CD3+ T cells was only due to an increase in CD8+ $\mathrm{T}$ cells and not in CD4+ T cells, since the CD4+ cells remained low after the nadir due to ThCR (Figs. 3, 4, 5). B cells did not recover after ThCR either (Fig. 6).

Preoperatively, CD4+ T, NK and B cells were significantly lower in the neo group as compared to the control group $(P<0.001, P<0.001$, and $P=0.044$, respectively). In the neo group, the CD8+ T cells recovered sharply in the preoperative period after ThCR and showed to be the only lymphocyte subset that was at similar level preoperatively as the control group $(P=0.6)$.

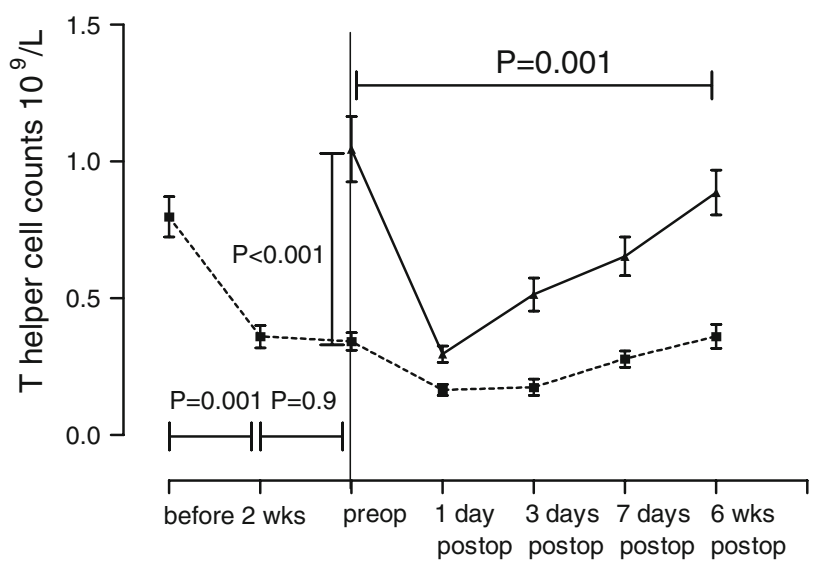

Fig. 3 CD3+CD4+ T cells counts (T helper cells) as determined by flow cytometry: patients with oesophageal cancer treated with (dashed line with filled square, neo) and without (solid line with filled triangle, controls) preoperative thermochemoradiation therapy. Values after 2 weeks of neoadjuvant therapy versus baseline values: $P=0.001$. Preoperative values versus values after 2 weeks of neoadjuvant therapy, $P=0.9$; neo versus controls, 1 day preoperatively, $P<0.001$. Between-group differences over time in the 6 weeks postoperative period; $P<0.001$

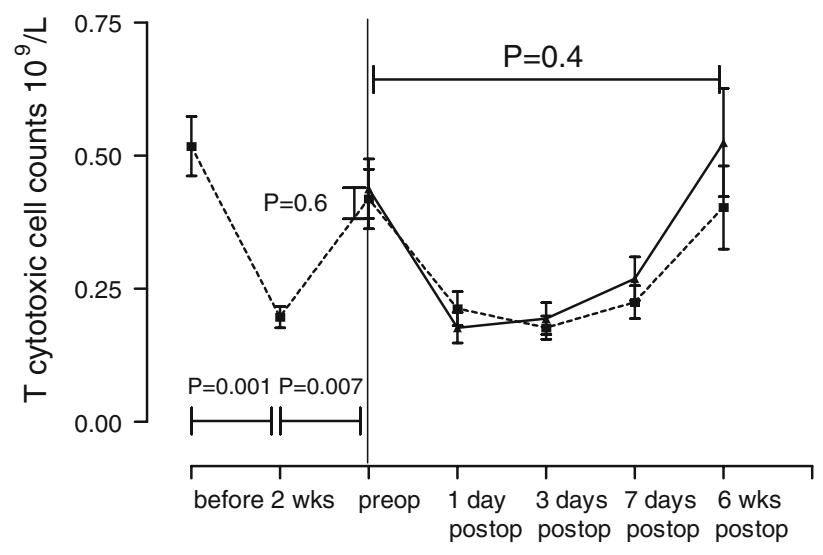

Fig. 4 CD3+CD8+ T cells counts (T cytotoxic cells) as determined by flow cytometry: patients with oesophageal cancer treated with (dashed line with filled square, neo) and without (solid line with filled triangle, controls) preoperative thermochemoradiation therapy. Values after 2 weeks of neoadjuvant therapy versus baseline values: $P=0.001$. Preoperative values versus values after 2 weeks of neoadjuvant therapy, $P=0.007$; neo versus controls, 1 day preoperatively, $P=0.6$. Between-group differences over time in the 6 weeks postoperative period; $P=0.4$

\section{Production of T lymphocyte-derived cytokines}

Baseline values of Th1-type cytokines (IL-2 and IFN- $\gamma$ ) and those of Th2-type cytokines (IL-4 and IL-13) in neo and control patients were comparable (Table 3 ).

After 2 weeks of ThCR supernatant levels of both Th1type cytokines (IL-2 and IFN- $\gamma$ ) and Th2-type cytokines (IL-4 and IL-13) were significantly decreased $(P=0.003$ and $P=0.011$ and $P=0.003$ and $P=0.013$, respectively, 


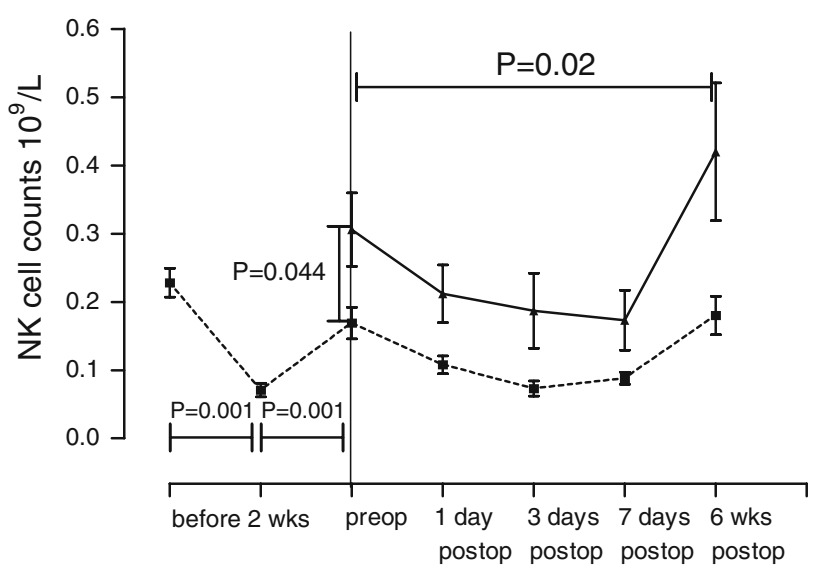

Fig. 5 CD16+CD56+ NK cell counts as determined by flow cytometry: Patients with oesophageal cancer treated with (dashed line with filled square, neo) and without (solid line with filled triangle, controls) preoperative thermochemoradiation therapy. Values after 2 weeks of neoadjuvant therapy versus baseline values: $P<0.001$. Preoperative values versus values after 2 weeks of neoadjuvant therapy, $P=0.001$; neo versus controls, 1 day preoperatively, $P=0.044$. Between-group differences over time in the 6 weeks postoperative period; $P=0.02$

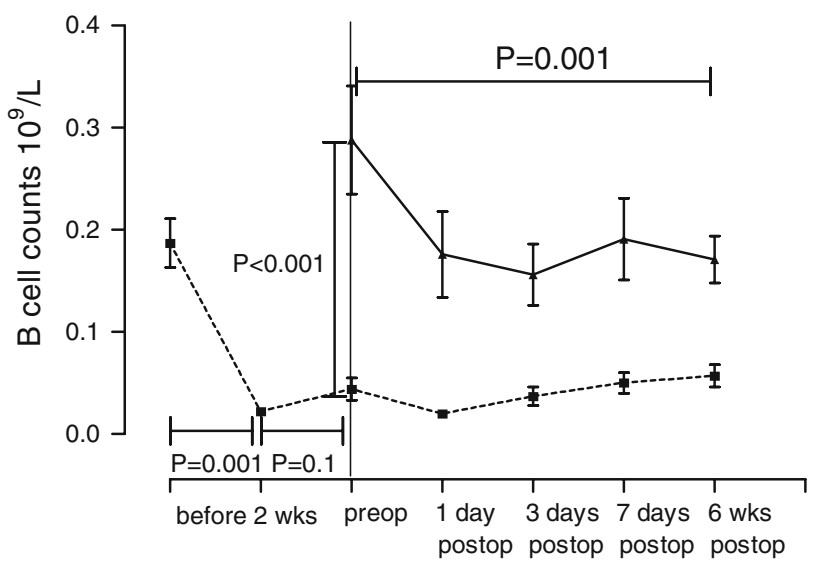

Fig. 6 CD19+ B cell counts as determined by flow cytometry: Patients with oesophageal cancer treated with (dashed line with filled square, neo) and without (solid line with filled triangle, controls) preoperative thermochemoradiation therapy. Values after 2 weeks of neoadjuvant therapy versus baseline values: $P<0.001$. Preoperative values versus values after 2 weeks of neoadjuvant therapy, $P=0.1$; neo versus controls, 1 day preoperatively, $P<0.001$. Between-group differences over time in the 6 weeks postoperative period; $P<0.001$

shown for IL-2 and IL-4 in Fig. 7a and 8a). Cytokine production per $\mathrm{T}$ lymphocyte (CD3+ cells) was not significantly decreased (Table 4, Fig. 7b, 8b). For Th2-type cytokine IL-13 even an increased production was found $(P=0.001$, Table 4) and also trendwise for IL-4 $(P=0.087$, Fig. 8b).

Preoperative absolute values (absolute values and per T lymphocyte) of both Th1-type cytokines and Th2-type cytokines were somewhat lower in the neo group compared to the control group, although not statistically significant.
Table 3 Absolute baseline values Th1- and Th2 cytokines

\begin{tabular}{llll}
\hline & Neo $(N=20)$ & Control $(N=12)$ & $P$ value* \\
\hline Th1 & & & \\
IL-2 & $3,388(191-11,438)$ & $2,909(197-9,046)$ & 0.525 \\
IFN $\gamma$ & $2,288(200-14,109)$ & $1,423(195-13,629)$ & 0.445 \\
Th2 & & & \\
IL-4 & $357(63-2,498)$ & $343(79-4,233)$ & 0.944 \\
IL-13 & $358(173-1,137)$ & $400(160-1,658)$ & 0.866 \\
\hline
\end{tabular}

Continuous variables are expressed as medians and ranges $(\mathrm{pg} / \mathrm{ml})$. Before neoadjuvant therapy (neo) and before operation (control)

Neo patients with preoperative treatment, control patients without preoperative treatment

* Mann-Whitney test (continuous variables)

Postoperative parameters of cellular immunity

\section{White blood cell and differential counts}

Granulocyte counts increased significantly after surgery in both groups $(P=0.008$ for neo and $P=0.005$ for control at postoperative day 1$)$, and decreased to preoperative levels. However, granulocytes were significantly lower at 6 weeks after surgery than at baseline in the neo patients $(P=0.04$, Fig. 1). The total leucocyte counts showed similar kinetics as for granulocytes for each of the groups (data not shown).

Surgery had no clear effect on monocytes in neo and control patients $(P=0.88$ for neo and $P=0.31$ for control patients, data not shown). In contrast, surgery induced a profound reduction in lymphocyte counts in both groups $(P<0.001$ for neo and $P=0.003$ for control patients, at postoperative day 1, Fig. 2). Both groups recovered to preoperative levels 6 weeks after operation, although neo patients did not reach baseline values $(P=0.003)$.

Between the two groups there was a significant difference over time postoperatively in leucocytes $(P=0.002)$, granulocytes $(P=0.012$, Fig. 1$)$ and lymphocytes $(P<0.001$, Fig. 2), with the lower values for the neo patients.

\section{Lymphocyte subsets}

Postoperative CD3+ T cell numbers decreased significantly in both groups $(P<0.001$ for neo patients and $P=0.003$ for control patients). Both groups recovered towards preoperative levels 6 weeks after operation, although neo patients did not reach baseline values ( $P=0.004$, data not shown). Similarly, CD4+ and CD8+ T cells showed a decrease after operation $(P<0.001$ and $P<0.001$, respectively, for neo patients; $P=0.003$ and $P=0.003$, respectively, for control patients; Figs. 3 , 4). In the neo patients, the observed low numbers of $\mathrm{CD} 4+\mathrm{T}$ cells after neoadjuvant treatment remained low during and after surgery, even after 6 weeks (Fig. 3). In contrast, CD8+ T cells recovered relatively fast 
(A)

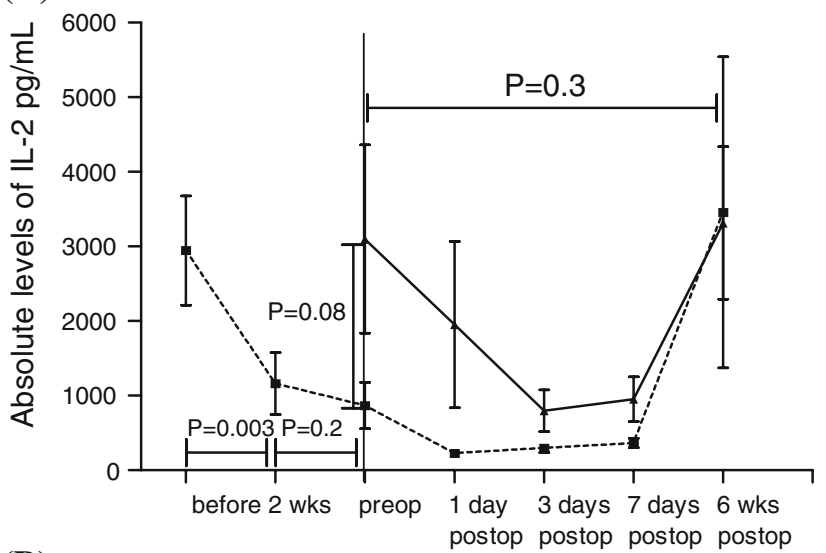

(B)

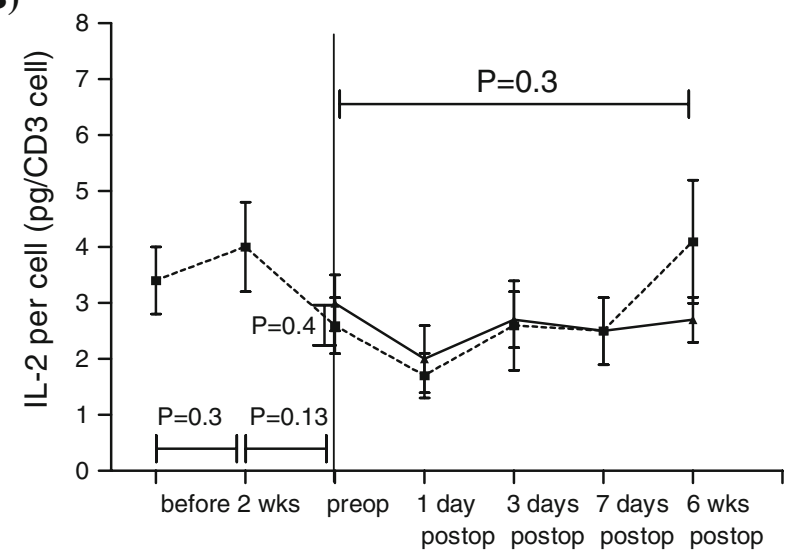

Fig. 7 a Supernatant levels of T helper cell type 1 (Th1) cytokine IL2 , expressed as absolute values in stimulated whole blood cultures from blood samples: Patients with oesophageal cancer treated with (dashed line with filled square, neo) and without (solid line with filled triangle, controls) preoperative thermochemoradiation therapy. Values after 2 weeks of neoadjuvant therapy versus baseline values: $P=0.003$. Preoperative values versus values after 2 weeks of neoadjuvant therapy, $P=0.2$; neo versus controls, 1 day preoperatively, $P=0.08$. Between-group differences over time in the 6 weeks postoperative period; $P=0.3$. b Supernatant levels of Th1 cytokine IL-2 per cell (corrected for no. of T cells): Patients with oesophageal cancer treated with (dashed line with filled square, neo) and without (solid line with filled triangle, controls) preoperative thermochemoradiation therapy. Values after 2 weeks of neoadjuvant therapy versus baseline values: $P=0.03$. Preoperative values versus values after 2 weeks of neoadjuvant therapy, $P=0.13$; neo versus controls, 1 day preoperatively, $P=0.4$. Between-group differences over time in the 6 weeks postoperative period; $P=0.3$

after the post-surgery nadir, just as was seen after neoadjuvant therapy (Fig. 4). Hence, there were no differences in preoperative and postoperative $\mathrm{CD} 8+\mathrm{T}$ cell counts between neo and control patients.

Similar to CD4+ T cell counts, also NK and B cell counts that first had decreased after neoadjuvant therapy were persistently low levels during and after surgery. In control patients, NK and B cell counts indeed decreased after surgery but still remained significantly higher than
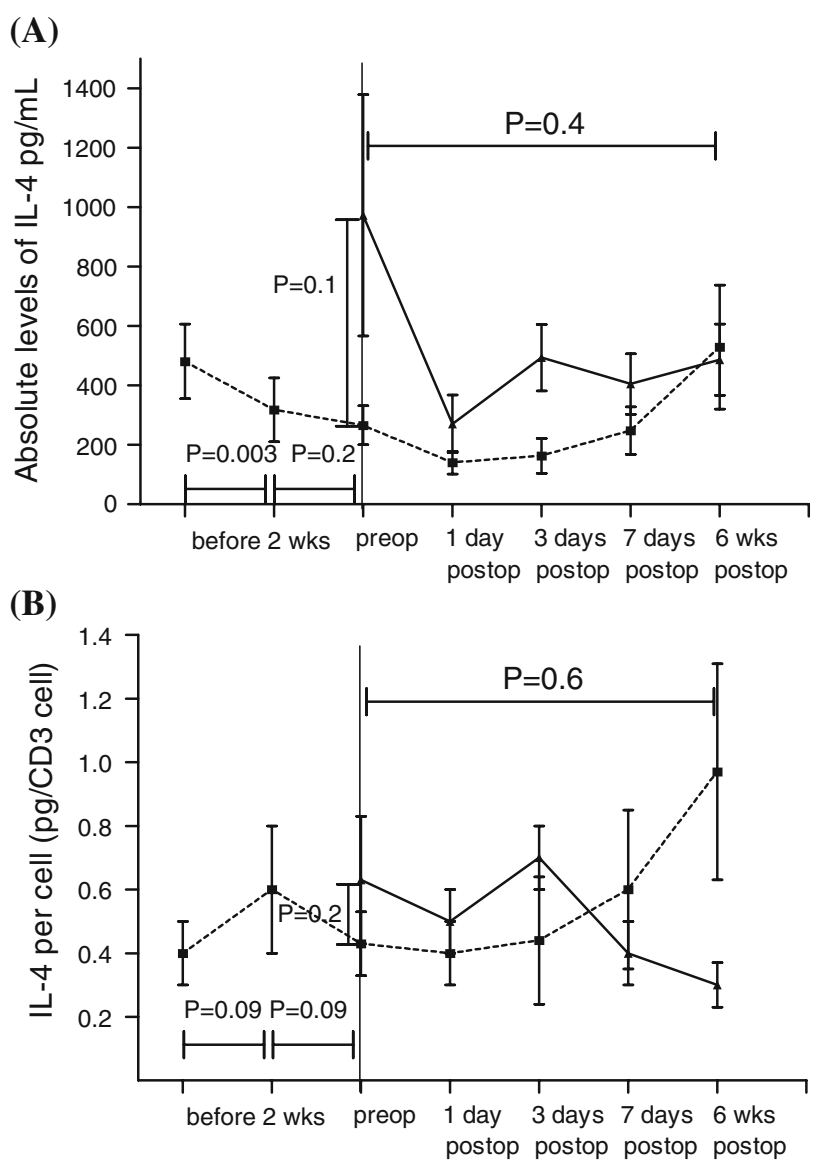

Fig. 8 a Supernatant levels of T helper cell type 2 (Th2) cytokine IL4, expressed as absolute values in stimulated whole blood cultures from blood samples: Patients with oesophageal cancer treated with (dashed line with filled square, neo) and without (solid line with filled triangle, controls) preoperative thermochemoradiation therapy. Values after 2 weeks of neoadjuvant therapy versus baseline values: $P=0.003$. Preoperative values versus values after 2 weeks of neoadjuvant therapy, $P=0.2$; neo versus controls, 1 day preoperatively, $P=0.1$. Between-group differences over time in the 6 weeks postoperative period; $P=0.4$. b Supernatant levels of Th2 cytokine IL-4 per cell (corrected for no. of T cells): Patients with oesophageal cancer treated with (dashed line with filled square, neo) and without (solid line with filled triangle, controls) preoperative thermochemoradiation therapy. Values after 2 weeks of neoadjuvant therapy versus baseline values: $P=0.009$. Preoperative values versus values after 2 weeks of neoadjuvant therapy, $P=0.09$; neo versus controls, 1 day preoperatively, $P=0.2$. Between-group differences over time in the 6 weeks postoperative period; $P=0.6$

counts in neo patients $(P=0.02$ and $P<0.001$, respectively, Figs. 5, 6).

\section{Production of T lymphocyte derived cytokines}

Absolute levels of Th1-type cytokines (IL-2 and IFN- $\gamma$ ) and Th2-type cytokines (IL-4 and IL-13) decreased significantly 1 day after operation in the neo group $(P<0.001$, $P=0.002, P=0.003$ and $P=0.002$, respectively). In the neo group cytokine production per T lymphocyte showed a 
Table 4 Values of Th1- and Th2-cytokines (corrected for number of T cells), in the 20 neo patients who received preoperative therapy

\begin{tabular}{llllll}
\hline & Baseline & After 2 weeks & $P$ value* & Preoperative & $P$ value* (vs. 2 weeks ThCR) \\
\hline Th1 & & & & & \\
IL-2 & $2.94(0.13-11.31)$ & $3.59(0.31-11.8)$ & 0.255 & $1.58(0.19-6.91)$ & 0.125 \\
IFN $\gamma$ & $1.79(0.14-10.54)$ & $1.15(0.21-10.54)$ & 0.605 & $0.52(0.20-7.14)$ & 0.053 \\
Th2 & & & & & 0.087 \\
IL-4 & $0.28(0.04-1.76)$ & $0.34(0.08-2.62)$ & 0.087 & $0.04-2.01)$ & 0.109 \\
IL-13 & $0.30(0.12-0.91)$ & $0.76(0.35-3.08)$ & 0.001 & $0.60(0.22-2.09)$ & \\
\hline
\end{tabular}

Neo, patients with preoperative treatment; continuous variables are expressed as medians and ranges (pg/CD3 cell); before neoadjuvant therapy (baseline), 2 weeks of neoadjuvant therapy and preoperative

* Wilcoxon (continuous variables)

comparable decrease in Th1- and Th2-type cytokine production after operation, however only IL-2 decreased significantly $(P=0.008$, see Fig. $7 b)$. In the control group there was a nonsignificant decrease in Th1- and Th2-type cytokine production per $\mathrm{T}$ lymphocyte.

At day 3 after surgery a clear recovery in production of IL-4 (absolute values and per T lymphocyte) was seen in the control group, while the neo group remained low (Fig. 8a, b). Six weeks after surgery graph lines of absolute levels of cytokines of both groups had merged and were by far similar. There was no significant between-group difference over time in both absolute and corrected levels of Th1and Th2-type cytokines (shown for IL-2 and IL-4 in Figs. 7, 8).

\section{Discussion}

Major surgery is known to be associated with immune suppression. Several studies, investigating alterations in immune defence after oesophageal resection, have shown that oesophagectomy depresses host immune response [41, 42]. However, little is known about the immunological effects of preoperative neoadjuvant treatment in these patients [40].

The present study describes a broad spectrum of both numerical and functional immunological parameters in a series of surgical patients, some of whom received preoperative treatment, while others were directly operated on. The study population was uniform in that all patients had potentially curable disease based on preoperative investigations, and clinical parameters were comparable in both groups.

In brief, patients who received neoadjuvant thermochemoradiation therapy demonstrated a significant decrease in the number of granulocytes and lymphocyte subsets. T cell cytokine release was decreased after 2 weeks of neoadjuvant therapy, while production per $\mathrm{T}$ helper cell remained the same or showed a modest increase. CD8+ (cytotoxic) $\mathrm{T}$ cells but not CD4+ (helper) $\mathrm{T}$ cells recovered in the period between neoadjuvant therapy and operation. CD4+ T (helper) cell cytokine production levels were almost recovered, preoperatively.

All lymphocyte subsets that had not recovered after neoadjuvant therapy (i.e., CD4+ T cells, NK and B cells but not CD8+ T cells) had persistent lower counts as compared to control patients (operated without neoadjuvant therapy) at 1 week postoperatively.

Hyperthermia causes a transient stimulation of the cellmediated immune response [38]. Recent research has shown that, when cancer cells are heated to approximately $41.6^{\circ} \mathrm{C}$, they form highly specific protein structures on their surface known as heat-shock proteins [27]. These proteins activate the patient's own NK cells to attack the cancer cells and stimulate the immune system [27]. Studies, evaluating the immune response after chemotherapy and/or radiation therapy, showed profound negative effects on the immune system [18, 22, 29, 36, 43]. In the present study, the neo patients underwent a combination of chemotherapy, radiotherapy and hyperthermia. The cumulative effects of this combination therapy led to a reduction of the white blood cell count which resulted in an overall reduced capacity to produce cytokines after 2 weeks of therapy. Apparently, the immunosuppressive impact of chemoradiation surpasses the immunostimulatory effect of hyperthermia.

In patients with sepsis, the loss of lymphocytes, contributing to immunosuppression by decreasing the number of available immune cells, is a well-known phenomenon. However, relatively recent work implicates that, not only a decrease in the number of effector cells, but also lymphocyte apoptosis plays a potential factor in the immunosuppression in sepsis [16]. The apoptotic cells appear to have important adverse effects on immune function by actively suppressing the inflammatory response [15]. It is unknown at present whether lymphocyte apoptosis plays a role in thermochemoradiation-induced immunosuppression, and may be an important subject for further study.

The differential recovery of CD8+ cells in contrast to CD4+ cells leading to a prolonged $\mathrm{T}$ cell imbalance after thermochemoradiation therapy has been described by 
others after intensive chemotherapy and after peripheral blood stem cell transplantation $[19,31]$. As most peripheral CD4+ en CD8+ T cells share a common primary developmental pathway until relatively late in their development, this significant difference in the rate of recovery is surprising. In short, $\mathrm{T}$ cell precursors move from the bone marrow to the thymus, and develop to $\mathrm{T}$ cells. In the thymus various $\mathrm{T}$ cell precursors can be distinguished by the expression of specific cell surface markers: first TCR ( $T$ cell receptor), second CD3 (which serves as the signal transduction component of TCR), and finally CD4/CD8. It could be hypothesised that the regeneration of both CD4+ and CD8+ T cells occurs exclusively via thymic dependent pathways [19]. However, there is no existing evidence to suggest that a suboptimal thymic regenerative capacity would preferentially affect CD4+ populations, which could explain the differential recovery rate $[3,32,37]$. Alternatively, extrathymic generation of CD8+ cells from haematopoietic precursors could be involved and/or peripheral expansion of mature CD8+ T cells, which remain in the secondary lymphoid tissues after completion of chemotherapy. Mackall et al. [19] suggested that extrathymic lymphopoiesis is involved, as in their study the predominance of CD8+ cells lacked CD28 expression. This lack of CD28 expression has been associated with impaired thymic function in HIV infection [4], post-bone marrow transplantation [2, 24], and aged hosts [34]. Expansions of CD8+CD57+ cells have also been described in combination with restricted $\mathrm{T}$ cell repertoire diversity [11]. Thus, the relatively rapid regeneration of CD8+ cells compared to the slow CD4+ regeneration might be due to thymic independent pathways that efficiently and selectively induce regeneration of distinct CD8+ subsets.

Furthermore, differences in sensitivity of the factors that regulate homoeostatic proliferation of memory CD8+ T cells as compared to memory CD4+ T cells may explain the slower recovery of the CD4+ $\mathrm{T}$ cell-population from thermochemoradiation. Homoeostatic proliferation of CD8+ T cells seems to depend on IL-15 and IL-7, whereas these cytokines are probably not required for homoeostatic proliferation of CD4+ T cells [39].

Further work is necessary to be able to specify the origin of regenerated CD8+ cells and either reject or sustain the explanation of biological distinctions between CD8+ and CD4+ T cell regenerative pathways. It may be of clinical importance to determine if a predominance of CD8+ cells exists of $\mathrm{CD} 8+\mathrm{CD} 28^{-}$and $\mathrm{CD} 8+\mathrm{CD} 57+$ subsets, since a limited effectiveness of these populations has been described in vivo [30]. A significant depression in cellmediated immunity in conjunction with possible alterations of CD8+ T cell subsets in neo patients as compared to control patients may have implications for the host defence against foreign pathogens as well as in the immunological control of disseminated tumour cells.

Although the role of the immune response in controlling tumour growth and cancer recurrence is controversial, there is evidence in oesophageal cancer suggesting that $\mathrm{T}$ cell infiltrates have a beneficial prognostic impact [14]. It was shown that $\mathrm{CD} 8+\mathrm{T}$ cell infiltrations within the tumour specimen have a favourable outcome [35]. And a recent research in patients with colorectal cancers indicated that type, density and location of immune cells within tumour tissues were a better predictor of patient survival than the current methods to stage colorectal cancers [10, 28]. Interestingly, colorectal tumours from patients without recurrence had higher immune cell densities of $\mathrm{CD} 3+$ cells within the tumour and at the infiltrating tumour margin. Among CD4+ T lymphocytes, Th1 and Th2 phenotypes can be distinguished [25]. Activation of these two functionally distinct subsets of mature $\mathrm{T}$ helper cells is of great importance for an effective immune response against infection. Th1 cells are mainly characterised by secretion of IL-2 and IFN- $\gamma$ and induce cell-mediated immune responses. Th2 cells preferentially secrete the cytokines IL-4 and IL-13 which favour humoural immune responses [7, 33]. Previously published results from our group showed that oesophagectomy severely depressed host immune responses. In the present study, neoadjuvant therapy caused both reduced $\mathrm{T}$ cell counts and decreased absolute levels of both Th1 and Th2 derived cytokines. This is in line with previous investigations, studying alterations in immune defence after neoadjuvant treatment [12]. However, in the present study it was shown that after neoadjuvant therapy production of the Th1 and Th2 cytokines per T helper cell was not decreased and for IL-13 even moderately increased, indicating that reduced cell counts rather than functional deficits contributed to the post-therapy reduction of $\mathrm{T}$ cell cytokine production.

At present, novel therapeutic strategies in oesophageal cancer mainly focus on applying new chemotherapeutic drugs, varying drug dosages, optimising the dose of radiotherapy or on adding hyperthermia to optimise long-term survival. However, the role of the host immunocompetence in the inhibition of disease progression and the way it is altered by the various new therapeutic strategies have only been partially investigated. The present study shows significant disturbances of host cellular immunity induced by preoperative thermochemoradiation compared to surgery alone in oesophageal cancer patients. This neoadjuvant therapy caused reduced T, NK and B cell counts, reduced overall Th1 and Th2 cytokine production, and differential recovery of $\mathrm{T}$ cytotoxic and $\mathrm{T}$ helper cells, leading to prolonged $\mathrm{T}$ cell imbalance that extends beyond the time of surgery. However, the study group was to small to conclude on the basis of the immune response whether to stop or continue 
neoadjuvant therapy, although it indicates that for individual patients it is worthwhile monitoring their immune status, having a vigilant attitude toward emerging infections. The oncological consequences of these immunological changes need further investigation, as recovery of $\mathrm{T}$ helper cytokine production towards surgery was less impaired than $\mathrm{T}$ helper cell counts.

Open Access This article is distributed under the terms of the Creative Commons Attribution Noncommercial License which permits any noncommercial use, distribution, and reproduction in any medium, provided the original author(s) and source are credited.

\section{References}

1. Albregts M, Hulshof MC, Zum Vorde Sive Vording PJ et al (2004) A feasibility study in oesophageal carcinoma using deep loco-regional hyperthermia combined with concurrent chemotherapy followed by surgery. Int J Hyperthermia 20:647-659

2. Autran B, Leblond V, Sadat-Sowti B et al (1991) A soluble factor released by CD8+CD57+ lymphocytes from bone marrow transplanted patients inhibits cell-mediated cytolysis. Blood 77:2237-2241

3. von Boehmer H, Kisielow P (1993) Lymphocyte lineage commitment: instruction versus selection. Cell 73:207-208

4. Brinchmann JE, Dobloug JH, Heger BH et al (1994) Expression of costimulatory molecule CD28 on T cells in human immunodeficiency virus type 1 infection: functional and clinical correlations. J Infect Dis 169:730-738

5. Brune IB, Wilke W, Hensler T et al (1999) Downregulation of T helper type 1 immune response and altered pro-inflammatory and anti-inflammatory $\mathrm{T}$ cell cytokine balance following conventional but not laparoscopic surgery. Am J Surg 177:55-60

6. Decker D, Schondorf M, Bidlingmaier F et al (1996) Surgical stress induces a shift in the type-1/type-2 T-helper cell balance, suggesting down-regulation of cell-mediated and up-regulation of antibody-mediated immunity commensurate to the trauma. Surgery 119:316-325

7. Del Prete G, De Carli M, Almerigogna F et al (1993) Human IL10 is produced by both type 1 helper (Th1) and type 2 helper (Th2) $\mathrm{T}$ cell clones and inhibits their antigen-specific proliferation and cytokine production. J Immunol 150:353-360

8. Dijk van JDP, Gonzalez Gonzalez D, Blank LECM (eds) (1989) Deep local hyperthermia with a four aperture array system of large waveguide radiators: results of simulation and clinical application. Hyperthermic oncology, vol 1. Taylor \& Francis, London, pp 573575

9. Earlam R, Cunha-Melo JR (1980) Oesophageal squamous cell carcinomas: II. A critical view of radiotherapy. Br J Surg 67:457-461

10. Galon J, Costes A, Sanchez-Cabo F et al (2006) Type, density, and location of immune cells within human colorectal tumors predict clinical outcome. Science 313:1960-1964

11. Gorochov G, Debre P, Leblond V et al (1994) Oligoclonal expansion of CD8+CD57+ T cells with restricted T-cell receptor beta chain variability after bone marrow transplantation. Blood 83:587-595

12. Heidecke CD, Weighardt H, Feith M et al (2002) Neoadjuvant treatment of esophageal cancer: immunosuppression following combined radiochemotherapy. Surgery 132:495-501

13. Hensler T, Hecker H, Heeg K et al (1997) Distinct mechanisms of immunosuppression as a consequence of major surgery. Infect Immun 65:2283-2291
14. Hosch SB, Meyer AJ, Schneider C et al (1997) Expression and prognostic significance of HLA class I, ICAM-1, and tumor-infiltrating lymphocytes in esophageal cancer. J Gastrointest Surg $1: 316-323$

15. Hotchkiss RS, Osmon SB, Chang KC et al (2005) Accelerated lymphocyte death in sepsis occurs by both the death receptor and mitochondrial pathways. J Immunol 174:5110-5118

16. Hotchkiss RS, Tinsley KW, Swanson PE et al (2001) Sepsis-induced apoptosis causes progressive profound depletion of $\mathrm{B}$ and CD4+ T lymphocytes in humans. J Immunol 166:69526963

17. Iyer R, Wilkinson N, Demmy T, Javle M (2004) Controversies in the multimodality management of locally advanced esophageal cancer: evidence-based review of surgery alone and combinedmodality therapy. Ann Surg Oncol 11:665-673

18. Kempf RA, Mitchell MS (1985) Effects of chemotherapeutic agents on the immune response: II. Cancer Invest 3:23-33

19. Mackall CL, Fleisher TA, Brown MR et al (1997) Distinctions between $\mathrm{CD} 8+$ and $\mathrm{CD} 4+\mathrm{T}$-cell regenerative pathways result in prolonged T-cell subset imbalance after intensive chemotherapy. Blood 89:3700-3707

20. Mafune K, Tanaka Y (2000) Influence of multimodality therapy on the cellular immunity of patients with esophageal cancer. Ann Surg Oncol 7:609-616

21. Mandard AM, Dalibard F, Mandard JC et al (1994) Pathologic assessment of tumor regression after preoperative chemoradiotherapy of esophageal carcinoma: clinicopathologic correlations. Cancer 73:2680-2686

22. Mantovani A, Luini W, Peri G et al (1978) Effect of chemotherapeutic agents on natural cell-mediated cytotoxicity in mice. J Natl Cancer Inst 61:1255-1261

23. Medical Research Council Oesophageal Cancer Working (2002) Surgical resection with or without preoperative chemotherapy in oesophageal cancer: a randomised controlled trial. Lancet 359:1727-1733

24. Moses RD, Sharrow SO, Stephany DA et al (1989) Cardiac allograft survival across major histocompatibility complex barriers in the rhesus monkey following $\mathrm{T}$ lymphocyte-depleted autologous marrow transplantation. IV: Immune reconstitution. Transplantation 48:774-781

25. Mosmann TR, Coffman RL (1989) TH1 and TH2 cells: different patterns of lymphokine secretion lead to different functional properties. Annu Rev Immunol 7:145-173

26. Muller JM, Erasmi H, Stelzner M et al (1990) Surgical therapy of oesophageal carcinoma. Br J Surg 77:845-857

27. Ostapenko VV, Tanaka H, Miyano M et al (2005) Immune-related effects of local hyperthermia in patients with primary liver cancer. Hepatogastroenterology 52:1502-1506

28. Pages F, Berger A, Camus M et al (2005) Effector memory T cells, early metastasis, and survival in colorectal cancer. N Engl J Med 353:2654-2666

29. Petrini B, Wasserman J, Blomgren H (1985) T-cell subsets in patients treated with pelvic irradiation for cancer. J Clin Lab Immunol 17:147-148

30. Posnett DN, Sinha R, Kabak S, Russo C (1994) Clonal populations of $\mathrm{T}$ cells in normal elderly humans: the T cell equivalent to "benign monoclonal gammapathy. J Exp Med 179:609-618

31. Rick O, Beyer J, Schwella N, Siegert W (2002) Influence of amifostine on reconstitution of lymphocyte subpopulations after conventional- and high-dose chemotherapy in patients with germ cell tumor. Ann Hematol 81:717-722

32. Robey E, Axel R (1990) CD4: collaborator in immune recognition and HIV infection. Cell 60:697-700

33. Romagnani S (1994) Lymphokine production by human $\mathrm{T}$ cells in disease states. Annu Rev Immunol 12:227-257 
34. Sansoni P, Cossarizza A, Brianti V et al (1993) Lymphocyte subsets and natural killer cell activity in healthy old people and centenarians. Blood 82:2767-2773

35. Schumacher K, Haensch W, Roefzaad C, Schlag PM (2001) Prognostic significance of activated CD8(+) T cell infiltrations within esophageal carcinomas. Cancer Res 61:3932-3936

36. Stjernsward J, Jondal M, Vanky F et al (1972) Lymphopenia and change in distribution of human $\mathrm{B}$ and $\mathrm{T}$ lymphocytes in peripheral blood induced by irradiation for mammary carcinoma. Lancet 1:1352-1356

37. Suzuki H, Punt JA, Granger LG, Singer A (1995) Asymmetric signaling requirements for thymocyte commitment to the CD4+ versus CD8+ T cell lineages: a new perspective on thymic commitment and selection. Immunity 2:413-425

38. Szmigielski S, Sobczynski J, Sokolska G et al (1991) Effects of local prostatic hyperthermia on human $\mathrm{NK}$ and $\mathrm{T}$ cell function. Int $\mathrm{J}$ Hyperthermia 7:869-880

39. Tan JT, Ernst B, Kieper WC et al (2002) Interleukin (IL)-15 and IL-7 jointly regulate homeostatic proliferation of memory phenotype CD8+ cells but are not required for memory phenotype CD4+ cells. J Exp Med 195:1523-1532
40. Tsutsui S, Sonoda K, Sumiyoshi K et al (1996) Prognostic significance of immunological parameters in patients with esophageal cancer. Hepatogastroenterology 43:501-509

41. Van Sandick JW, Boermeester MA, Gisbertz SS et al (2003) Lymphocyte subsets and T(h) $1 / T(h) 2$ immune responses in patients with adenocarcinoma of the oesophagus or oesophagogastric junction: relation to pTNM stage and clinical outcome. Cancer Immunol Immunother 52:617-624

42. Van Sandick JW, Gisbertz SS, Ten Berge IJ et al (2003) Immune responses and prediction of major infection in patients undergoing transhiatal or transthoracic esophagectomy for cancer. Ann Surg 237:35-43

43. Verastegui EL, Morales RB, Barrera-Franco JL et al (2003) Longterm immune dysfunction after radiotherapy to the head and neck area. Int Immunopharmacol 3:1093-1104

44. Wieder HA, Brucher BL, Zimmermann F et al (2004) Time course of tumor metabolic activity during chemoradiotherapy of esophageal squamous cell carcinoma and response to treatment. J Clin Oncol 22:900-908 